\title{
Simulationstraining in der Weiterbildung - Einsatz von virtuellen laparoskopischen Operations- simulatoren in einem chirurgischen Trainingskurs
}

\author{
Simulation Training in Surgical Education - Application of Virtual Reality \\ Laparoscopic Simulators in a Surgical Skills Course
}

Autoren

Institut
K. S. Lehmann, J. Gröne, J. C. Lauscher, J.-P. Ritz, C. Holmer, U. Pohlen, H.-J. Buhr

Charité - Campus Benjamin Franklin, Chirurgische Klinik I, Berlin, Deutschland

Schlüsselwörter
Simulator
laparoskopische Chirurgie
Ausbildungsoperation
virtuelles OP-Training
Training
Lernkurve
Key words
simulator
laparoscopic surgery
training operation
virtual surgery training
training
learning curve

Bibliografie

DOI http://dx.doi.org/ 10.1055/s-0031-1283984 Zentralbl Chir 2012; 137: 130-137 (c) Georg Thieme Verlag KG Stuttgart · New York ISSN 0044-409X

\section{Korrespondenzadresse} Dr. med. Kai Siegfried Lehmann Charité - Campus Benjamin Franklin, Chirurgische Klinik I Hindenburgdamm 30 12203 Berlin Deutschland Tel.: +49/ $30 / 84452543$ Fax: $+49 / 30 / 84452740$ kai.lehmann@charite.de

\section{Zusammenfassung}

V

Hintergrund: Training und Simulation spielen eine zunehmende Rolle in der chirurgischen Ausbildung. Virtuelle OP-Simulatoren bieten heute anspruchsvolle laparoskopische Trainingsszenarien und detaillierte Bewertungsmöglichkeiten. Damit bieten sich die Geräte auch zur Verwendung in chirurgischen Trainingskursen an. Ziel der vorliegenden Studie war es, die Eignung eines OP-Simulators zum Training und zur Leistungsbewertung in einem etablierten chirurgischen Trainingskurs zu untersuchen.

Material und Methoden: Die Studie wurde während des „Praktischen Kurses für Viszeralchirurgie“ in Warnemünde durchgeführt. 36 von 108 Kursteilnehmer wurden zufällig für die Teilnahme ausgewählt. Die Teilnehmer trainierten über 15 Trainingssessions in 5 Tagen an 4 identischen OP-Simulatoren (LapSim) mit 2 standardisierten Übungen. 16 Trainingsparameter und 2 errechnete Scores wurden erfasst. Mit Fragebögen wurden die laparoskopische Erfahrung, die Ausbildungssituation und die Akzeptanz der Simulatoren erfragt. Die Daten wurden mit nicht-parametrischen Tests analysiert, zudem wurde eine Subgruppenanalyse nach laparoskopischer Erfahrung zur Beurteilung der Konstruktvalidität durchgeführt.

Ergebnisse: Das Alter der Teilnehmer lag bei 32 (27-41) Jahren, die Berufserfahrung bei 3(111) Jahren. Am Simulator zeigten sich typische laparoskopische Lernkurven mit initialer signifikanter Verbesserung der Scores und nachfolgender Plateauphase über 5 Trainingstage. Es konnten rhythmische Leistungsschwankungen für die Einzelsessions gemessen werden, wobei ein verkürzter Nachtschlaf eine deutliche Leistungseinschränkung nach sich zog. Die unterschiedliche laparoskopische Erfahrung der Teilnehmer konnte signifikant differenziert werden $(\leq 20$ vs. $>20$ laparoskopische Operationen; $\mathrm{p} \leq 0,001)$. Die Be-

\section{Abstract \\ $\nabla$}

Background: Training and simulation are gaining importance in surgical education. Today, virtual reality surgery simulators provide sophisticated laparoscopic training scenarios and offer detailed assessment methods. This also makes simulators interesting for the application in surgical skills courses. The aim of the current study was to assess the suitability of a virtual surgery simulator for training and assessment in an established surgical training course.

Materials and Methods: The study was conducted during the annual "Practical Course for Visceral Surgery" (Warnemuende, Germany). 36 of 108 course participants were assigned at random for the study. Training was conducted in 15 sessions over 5 days with 4 identical virtual surgery simulators (LapSim) and 2 standardised training tasks. The simulator measured 16 individual parameters and calculated 2 scores. Questionnaires were used to assess the test persons' laparoscopic experience, their training situation and the acceptance of the simulator training. Data were analysed with non-parametric tests. A subgroup analysis for laparoscopic experience was conducted in order to assess the simulator's construct validity and assessment capabilities.

Results: Median age was 32(27-41) years; median professional experience was 3 $(1-11)$ years. Typical laparoscopic learning curves with initial significant improvements and a subsequent plateau phase were measured over 5 days. The individual training sessions exhibited a rhythmic variability in the training results. A shorter night's sleep led to a marked drop in performance. The participants' different experience levels could clearly be discriminated ( $\leq 20$ vs. $>20$ laparoscopic operations; $\mathrm{p} \leq 0.001$ ). The questionnaire showed that the majority of the participants had limited training opportunities in their hospitals. The simulator training was very well accepted. However, 
fragung zeigte, dass die Mehrzahl der Teilnehmer in Ihren Kliniken kaum Trainingsmöglichkeiten hatte. Die Akzeptanz des Simulatortrainings war sehr hoch, allerdings schätzten die Teilnehmer die tatsächlichen Kosten der Geräte wesentlich zu niedrig ein.

Schlussfolgerung: Die Lernkurve am Simulator konnte während des Kurses erfolgreich durchlaufen werden. Die Konstruktvalidität wurde im Kursrahmen nachgewiesen. Das verwendete Bewertungssystem kann zur laparoskopischen Leistungsbewertung in Trainingskursen beitragen. Die Akzeptanz des Simulatortrainings ist hoch, die Kosten der Simulatoren sind zur Verwendung in einem großen Trainingskurs derzeit jedoch zu hoch. the participants severely misjudged the real costs of the simulators that were used.

Conclusions: The learning curve on the simulator was successfully mastered during the course. Construct validity could be demonstrated within the course setting. The simulator's assessment system can be of value for the assessment of laparoscopic training performance within surgical skills courses. Acceptance of the simulator training is high. However, simulators are currently too expensive to be used within a large training course.

\section{Einleitung}

Die praktische Weiterbildung von Chirurgen hat sich in den letzten Jahrzehnten deutlich gewandelt. Während die praktische Ausbildung früher überwiegend in Rahmen einer traditionellen Lehrer-Schüler-Beziehung im OP stattfand, kommt heute der Simulation und dem Training vor Eintritt in den OP eine immer größere Bedeutung zu. Mit der Einführung der minimalinvasiven Chirurgie seit den späten 1980er Jahren ist zudem ein erheblicher zusätzlicher Bedarf an speziellen Trainingsprogrammen entstanden $[1,2]$. In den letzten zwei Jahrzehnten wurden deshalb computerbasierte chirurgische Operationssimulatoren (Virtual Reality Simulator, VRS) entwickelt, die ein virtuelles minimal-invasives Training am Computer erlauben. Die ersten Geräte gestatteten aufgrund der verfügbaren Computerleistung nur abstrahierte Übungen in schematischen 3D-Welten. Die exponentielle Entwicklung der Computerleistung in den letzten Jahren hat jedoch dazu geführt, dass heute anspruchsvolle Simulationen realisierbar sind.

Virtuelle OP-Simulatoren bestehen üblicherweise aus einem Computer mit leistungsfähiger Grafikkarte. Eine firmenspezifische Software stellt die OP-Simulation auf einem Monitor dar. Hinzu kommen Eingabegeräte, die laparoskopische Instrumente nachahmen. Analog zum Flugsimulator können mit OP-Simulatoren prinzipiell beliebige Szenarien dargestellt und trainiert werden. Laparoskopische Basisübungen werden von allen Geräten angeboten. Hierbei handelt es sich u. a. um das Training von Kamera- und Instrumentenführung, die ein- oder beidhändige Manipulation von Objekten, sowie Anwendung spezifischer Techniken wie zum Beispiel die Applikation von Clips. Fortgeschrittene Übungen wie z.B. vollständige Cholezystektomien werden derzeit nur von einigen Simulatoren angeboten. Prinzipiell wird dabei eine echte Simulation angestrebt, also eine freie Interaktion mit der simulierten Umgebung. Tatsächlich sind die Interaktionsmöglichkeiten jedoch noch begrenzt, was insbesondere die Interaktion mit Weichgewebe betrifft. Vollständige OP-Szenarien sind daher in ihrem Realismus noch deutlich eingeschränkt. Allerdings ist es fraglich, ob vollständige OP-Szenarien für das Training tatsächlich erforderlich sind, da mit den vorhandenen - teilweise abstrakten - Übungsszenarien spezifische manuelle und koordinatorische Fähigkeiten gezielt trainiert werden können.

Gegenüber den konventionellen Trainingsmethoden wie z.B. dem Pelvitrainer zeichnen sich virtuelle OP-Simulatoren insbesondere auch durch die automatisierte Leistungserfassung aus. OP-Simulatoren können eine Vielzahl motorischer, koordinativer und operationsspezifischer Trainingsparameter automatisch erheben und abspeichern. Hiermit besteht erstmals die Möglich- keit, eine Bewertung des chirurgischen Trainings standardisiert und einfach umsetzbar durchzuführen.

Virtuelle laparoskopische OP-Simulatoren werden in Deutschland bislang nur zögerlich angenommen, international jedoch zunehmend eingesetzt [3]. Neben der immer noch eingeschränkten Akzeptanz gegenüber der neuen Technik sind auch finanzielle Aspekte bedeutsam. Die Geräte sind mit Anschaffungspreisen von ca. $50000 €$ bis $150000 €$ verhältnismäßig teuer. OP-Simulatoren wurden umfangreich wissenschaftlich evaluiert und in Bezug auf ihre Validität untersucht $[4,5]$. Hierbei konnte insbesondere auch nachgewiesen werden, dass ein Transfer von erlernten Fähigkeiten in den OP stattfindet [6, 7]. Mit diesen Möglichkeiten bieten sich OP-Simulatoren auch zur Verwendung in chirurgischen Trainingskursen an. Die potenziellen Vorteile beinhalten Trainingsszenarien, die nur von Simulatoren dargestellt werden können, und eine erhöhte Motivation für Kursteilnehmer. Zudem erlauben OP-Simulatoren eine Leistungsbewertung, die für das konventionelle Training kaum oder nur mit hohem Aufwand umsetzbar ist. Hiermit wäre eine Evaluation der Trainingsleistung im Rahmen eines OP-Kurses leicht durchführbar.

Ziel der vorliegenden Studie war es daher, den Einsatz von OP-Simulatoren in einem etablierten chirurgischen Trainingskurs zu untersuchen. Zielkriterien waren a) die Darstellung von Lernkurven am OP-Simulator während des Kursverlaufes, b) die Untersuchung von Einzelparametern und errechneten Scores zur Leistungsbewertung und c) die Evaluation der Praktikabilität und Akzeptanz des Simulatortrainings bei den Kursteilnehmern. Mit diesen Fragestellungen sollte die Eignung von OP-Simulatoren für die strukturierte chirurgische Weiterbildung im Rahmen von OP-Kursen näher definiert werden. Die Studie wurde während des „Praktischen Kurses für Viszeralchirurgie“ in Warnemünde durchgeführt. Dieser Kurs ist seit mehr als zehn Jahren etabliert und weist mit derzeit 108 Teilnehmern je Kurs eine hohe Teilnehmerzahl auf.

\section{Material und Methoden}

\section{$\nabla$}

Die Studie wurde während des „Praktischen Kurses für Viszeralchirurgie" in Warnemünde durchgeführt. Der WarnemündeKurs gehört zu den am häufigsten besuchten chirurgischen Trainingskursen in Deutschland [8]. Der Kurs besteht aus einem 7-tägigen Programm mit konventionellen und laparoskopischen viszeralchirurgischen Übungen an Tierpräparaten und im Tier-OP [9]. Der Kurs wird von mehreren chirurgischen Fakultäten (Berlin, Dresden, Rostock, Freiburg, München) organisiert und hat eine standardisierte chirurgische Ausbildung zum Ziel. Das Pro- 
gramm umfasst einen ersten Teil (Tage 1 -4) mit Übungen für die konventionelle Chirurgie und einen zweiten Teil (Tage 5-7) mit laparoskopischen Übungen.

\section{Studiendesign}

Für die Studie wurde der virtuelle laparoskopische Operationssimulator LapSim (Surgical Science, Göteborg, Schweden) benutzt. Dieser Simulator ist einer der am meisten benutzten und validierten Operationssimulatoren $[4,10,11]$. LapSim besteht aus einem Laptop-Computer mit speziellen Eingabegeräten, die laparoskopische Instrumente nachbilden $(\boldsymbol{A} \mathbf{A b b} .1 \mathbf{a})$. Details zu diesem Simulator wurden bereits ausführlich beschrieben $[12,13]$. Für die Studie wurden vier identische Simulatoren benutzt.

Zwei standardisierte Simulatorübungen wurden für die Studie ausgewählt:

1.) „Lifting \& Grasping“ ( $\bullet$ Abb. 1 b): Mit dieser Übung wurde die Instrumenten-Zielführung und einfache bimanuelle Objektinteraktion trainiert. Es wurde eine abstrakte Szene dargestellt, in der eine chirurgische Nadel unter einem Quader verborgen war. Der Proband musste den Quader mit einem Instrument anheben und die Nadel mit dem anderen Instrument ergreifen. Die Nadel musste dann in einem definierten Zielgebiet abgelegt werden. Die Aufgabe wurde mit verschiedenen räumlichen Anordnungen wiederholt. Berührungen des Hintergrundes wurden als Fehler gewertet und als Gewebeschädigung (TiDam) bzw. maximale Gewebeschädigung (MaDam) erfasst. Der Simulator speicherte automatisch sieben Parameter und bildete daraus einen Score ( $\bullet$ Tab.1). Der Schwierigkeitsgrad der Übung wurde hoch eingestellt (Objekte links / rechts: Je 3; Objektgröße: $10 \mathrm{~mm}$; Zielgebietgröße: $20 \mathrm{~mm}$, Timeout: $10 \mathrm{~s}$ ).

2.) „Clip Applying“ ( $\bullet$ Abb.1 c): Diese Übung trainierte eine komplexe bimanuelle Objektinteraktion. Ziel war es, ein simuliertes Gefäß mit Clips zu verschließen und dann zu durchtrennen. Bei

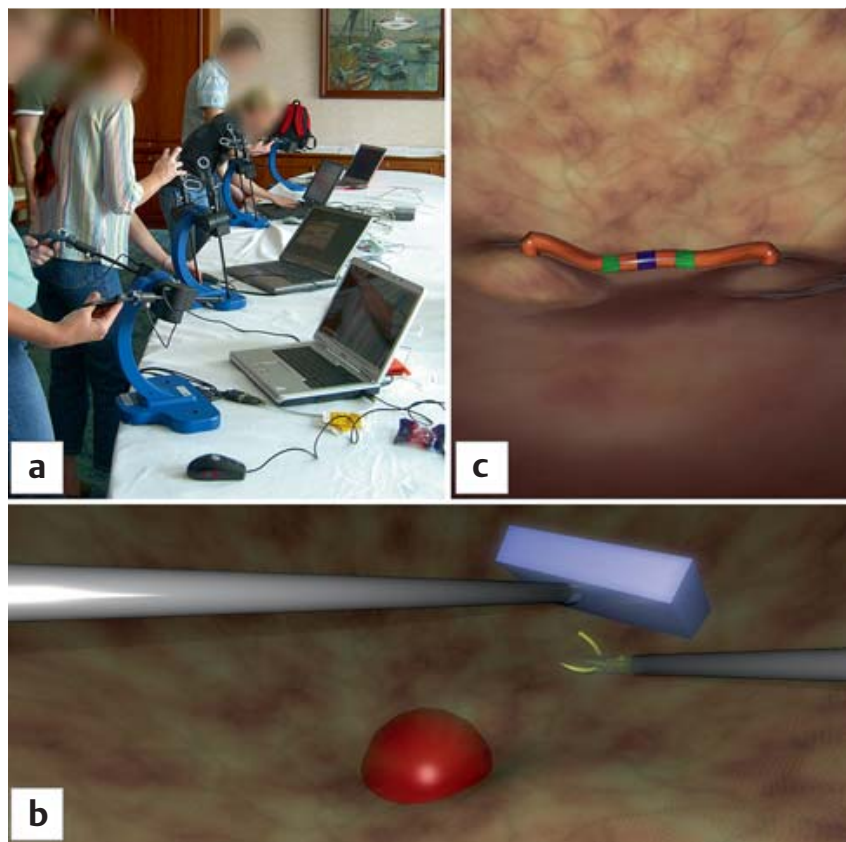

Abb. 1 Simulatorübungen. a Einsatz von vier identischen LapSim-Simulatoren. b Übung „Clip Applying“. c Übung „Lifting \& Grasping“.

zu starkem Zug auf das Gefäß zerriss dieses und löste eine Blutung aus, die ebenfalls mit einem Clip zu stillen war. Schwierig war hierbei insbesondere die fehlende haptische Rückkopplung der auf das Gefäß einwirkenden Zugkräfte. Dies entspricht der realen Laparoskopie mit eingeschränkter Haptik und überwiegend optischer Kontrolle der Interaktion. Dies erfordert von Anfängern in der minimal-invasiven Chirurgie ein erhebliches Umdenken im Vergleich zur konventionellen Chirurgie. Der Simu-

Tab. 1 Scores und Einzelparameter für die Simulatorübungen. Die Einzelparameter werden vom Simulator automatisch erhoben und gespeichert. Die Scores werden aus den Einzelparametern errechnet. Pfadlänge (RIPL, LIPL) und Winkelpfad (RIAP, LIAP) messen den zurückgelegten Weg der Instrumentenspitze, bzw. die Winkelbewegungen des Instrumentes. Beide Parameter beschreiben die Bewegungsökonomie. Verwendete Abkürzungen: LIPL: left instrument path length, LIAP: left instrument angular path, RIPL: right instrument path length, RIAP: right instrument angular path, TiDam: tissue damage, MaDam: maximum damage, BPC: badly placed clips, DRC: dropped clips, MaStretch: maximum stretch damage, BLS: blood loss.

\begin{tabular}{|c|c|c|c|}
\hline Parameter & Abkürzung & Einheit & Beschreibung \\
\hline \multicolumn{4}{|l|}{ Lifting \& Grasping (LG) } \\
\hline Score & Score & keine & $\begin{array}{l}\text { Score aus den Einzelparametern mit einer Gewichtung } \\
\text { von jeweils } 14,14 \%\end{array}$ \\
\hline Übungszeit & Zeit & Sekunden & Zeit für alle Ziele \\
\hline linkes Instrument, Pfadlänge & LIPL & Meter & zurückgelegte Länge der Instrumentenspitze \\
\hline linkes Instrument, Winkelpfad & LIAP & Grad & Winkelbewegungen des Instrumentes \\
\hline rechtes Instrument, Pfadlänge & RIPL & Meter & s. LIPL \\
\hline rechtes Instrument, Winkelpfad & RIAP & Grad & S. LIAP \\
\hline Gewebeschädigung & TiDam & Anzahl & Anzahl der unerwünschten Gewebekontakte \\
\hline maximale Gewebeschädigung & MaDam & Millimeter & maximale Eindrucktiefe bei unerwünschtem Gewebekontakt \\
\hline \multicolumn{4}{|l|}{ Clip Applying (CA) } \\
\hline Score & Score & keine & $\begin{array}{l}\text { Score aus den Einzelparametern mit einer Gewichtung von } \\
20 \% \text { für Blutverlust und jeweils } 10 \% \text { für alle anderen Parameter }\end{array}$ \\
\hline Übungszeit & Zeit & Sekunden & s. LG Zeit \\
\hline linkes Instrument, Pfadlänge & LIPL & Meter & s. LG LIPL \\
\hline linkes Instrument, Winkelpfad & LIAP & Grad & S. LG LIAP \\
\hline rechtes Instrument, Pfadlänge & RIPL & Meter & s. LG RIPL \\
\hline rechtes Instrument, Winkelpfad & RIAP & Grad & s. LG RIAP \\
\hline nicht korrekt platzierte Clips & $\mathrm{BPC}$ & Anzahl & Clips, die nicht korrekt platziert wurden \\
\hline verlorene Clips & DRC & Anzahl & Clips, die im Situs fallen gelassen wurden \\
\hline maximale Gewebedehnung & MaStretch & Prozent & Schädigung des Gefäßes durch Zug \\
\hline Blutverlust & BLS & Milliliter & Blutverlust \\
\hline
\end{tabular}


lator erfasste automatisch neun Parameter und bildete daraus einen Score ( $\bullet$ Tab. 1). Der Schwierigkeitsgrad der Übung wurde hoch eingestellt (Clipgebietgröße: 2; Schnittgebietgröße: 2; Sensitivität der Zugschädigung: Hoch; Blutfluss: 21/ min).

Alle 108 Teilnehmer des OP-Kurses wurden gebeten, auf einem initialen Fragebogen anzugeben, ob sie am Simulatortraining teilnehmen möchten. Von 98 Ja-Antworten wurden 36 Teilnehmer zufällig für die Teilnahme ausgewählt. Die Studie wurde während des 2. bis 6. Kurstages durchgeführt. Für beide Übungen waren jeweils 15 Trainingsdurchläufe (Sessions) vorgesehen. Um eine Integration der Studie in den Kursablauf mit knapper Zeitplanung zu ermöglichen, konnten die 15 Sessions variabel auf die 5 Studientage verteilt werden. Hierbei waren 3 Sessions je Tag vorgegeben; diese konnten bei Bedarf auf 2-4 Sessions / Tag bei insgesamt mindestens 4 Trainingstagen verteilt werden. Die Teilnehmer hatten keine Möglichkeit, außerhalb der Sessions am Simulator zu trainieren.

Die Teilnehmer wurden vor Beginn der Studie über den Ablauf der Übungen, die erfassten Parameter und die Fehlererfassung informiert. Jeder Teilnehmer konnte vor der ersten Session 5 min am Simulator ohne Datenerfassung trainieren. Mit dem Aushängen einer anonymen Rangliste wurde eine kompetitive Atmosphäre unterstützt.

\section{Fragebögen}

Vor Beginn der Studie wurden demographische Daten und Daten zur Berufserfahrung mit einem Fragebogen erhoben. Nach Abschluss der Studie wurden Fragen zur chirurgischen Ausbildung der Teilnehmer sowie zur Akzeptanz des Simulationstrainings mit einem zweiten Fragebogen gestellt. Die Antwortmöglichkeiten wurden jeweils mit einer 5-Punkte-Likert-Skala gegeben.

\section{Statistik}

Die Trainingsdaten wurden automatisch vom Simulator erhoben und wurden zusammen mit den Daten der Fragebögen anonymisiert in ein Statistikprogramm (SPSS 17, SPSS Inc., Chicago, USA) übertragen. Da aufgrund der Teilnehmerzahl nicht von einer Normalverteilung auszugehen war, werden kontinuierliche Variablen als Median (Minimum-Maximum) angegeben (Mittelwerte für Liniendiagramme). Kategoriale Variablen werden als Anzahl (Prozent) angegeben. Für Vergleiche zwischen unabhängigen Variabeln wurde der Mann-Whitney-U-Test benutzt, für Vergleiche zwischen abhängigen Variablen der Wilcoxon-Rang- summentest. Eine dichotome Subgruppenanalyse nach laparoskopischer Erfahrung wurde anhand der erhobenen demographischen Daten durchgeführt. Das Signifikanzniveau wurde auf 0,05 (zweiseitig) festgelegt.

\section{Ergebnisse}

Vor Beginn des Simulatortrainings wurden grundlegende demographische Angaben erfragt ( $\bullet$ Tab.2). Die Teilnehmer waren im Median mit 3 Berufsjahren keine Anfänger in der Chirurgie. Frauen und Männer waren annähernd gleich vertreten. Die überwiegende Anzahl der Teilnehmer kam aus Krankenhäusern der Grundversorgung. Anhand der Anzahl eigenständig durchgeführter Laparoskopien wurden die Teilnehmer in zwei Erfahrungsgruppen für die statistische Auswertung eingeteilt ( $\bullet$ Tab.2). In der Gruppe mit geringer Erfahrung ( $\leq 20$ eigenständige laparoskopische Operationen; $\mathrm{n}=21$; 2 Berufsjahre) hatten $81 \%$ weniger als 10 Laparoskopien selbst durchgeführt. In der Gruppe mit hoher Erfahrung (>20 eigenständige laparoskopische Operationen; $n=15$; 6 Berufsjahre) hatten zwei Drittel mehr als 50 Laparoskopien durchgeführt.

Jeder Teilnehmer trainierte im Median 9:11 Minuten pro Tag am Simulator (drei Übungssessions je Tag mit jeweils zwei Übungen). Die Scores zeigten eine signifikante Verbesserung von Tag $1 \mathrm{zu}$ Tag 2 (LG Score: $\mathrm{p} \leq 0,001$; CA Score: $\mathrm{p} \leq 0,001$ ) und von Tag 2 zu Tag 3 (LG Score: $p=0,014$; CA Score: $p=0,040$, $\bullet$ Abb. 2 ). Der weitere Leistungsanstieg war langsamer und zeigte keine signifikanten Unterschiede mehr.

- Abbildung 3 zeigt die den Scores zugrunde liegenden Einzelparameter des Simulators. Die Werte für die Einzelparameter wurden zu 100\% der Bestleistung aller Teilnehmer eines Tages berechnet, um eine gemeinsame Darstellung zu ermöglichen (Einzelparameter: Kleinere Werte=bessere Leistung; Scores: Größere Werte = bessere Leistung). Insgesamt zeigt sich für die meisten Parameter ein harmonischer Verlauf mit ähnlicher prozentualer Leistungsverbesserung über 5 Tage. Für die Lifting \& Grasping-Übung (LG) zeigte RIPL (Pfadlänge der rechten Hand als Ausdruck der Bewegungsökonomie) die höchsten Werte und den geringsten mittleren Abstand zur Bestleistung. Für diesen Parameter lag die geringste Leistungsvariabilität vor. Danach folgten die manuellen Parameter RIAP, LIPL und LIAP sowie die Übungszeit in engem Abstand. Mit deutlich weiterem Abstand

\begin{tabular}{|c|c|c|c|}
\hline & alle Teilnehmer & $\begin{array}{l}\leq 20 \text { lap. OPs } \\
\text { (geringe Erfahrung) }\end{array}$ & $\begin{array}{l}>20 \text { lap. OPs } \\
\text { (hohe Erfahrung) }\end{array}$ \\
\hline Anzahl, n (\%) & 36 & $21(58)$ & $15(42)$ \\
\hline Age, Jahre (min-max) & $32(27-41)$ & $30(27-36)$ & $35(31-41)$ \\
\hline Berufsjahre, Jahre (min - max) & $3(1-11)$ & $2(1-3)$ & $6(3-11)$ \\
\hline weibliches Geschlecht, n (\%) & $17(47,2)$ & $12(57)$ & $5(33)$ \\
\hline \multicolumn{4}{|l|}{ Krankenhaustyp, n (\%) } \\
\hline k. A. & $2(5,6)$ & 0 & $2(13)$ \\
\hline Grundversorgung & $16(44,4)$ & $9(43)$ & $7(47)$ \\
\hline Schwerpunktversorgung & $4(11,1)$ & $2(10)$ & $2(13)$ \\
\hline Maximalversorgung & $8(22,2)$ & $5(24)$ & $3(20)$ \\
\hline Universitätsklinik & $6(16,7)$ & $5(24)$ & $1(7)$ \\
\hline \multicolumn{4}{|c|}{ eigenstandige Laparoskopien, n (\%) } \\
\hline $0-3$ & $10(27,8)$ & $10(48)$ & - \\
\hline $4-10$ & $7(19,4)$ & $7(33)$ & - \\
\hline $11-20$ & $4(11,1)$ & $4(19)$ & - \\
\hline $21-50$ & $5(13,9)$ & - & $5(33)$ \\
\hline$>50$ & $10(27,8)$ & - & $10(67)$ \\
\hline
\end{tabular}

Tab. 2 Demografische Daten, Median (min - max) für kontinuierliche Variablen, n (\%) für kategoriale Variablen. Es wurden zwei Gruppen nach laparoskopischer Vorerfahrung gebildet (eigenständige laparoskopische Operationen; $\leq 20$ lap. OPs vs. > 20 lap. OPs), die für eine Subgruppenanalyse verwendet wurden. 


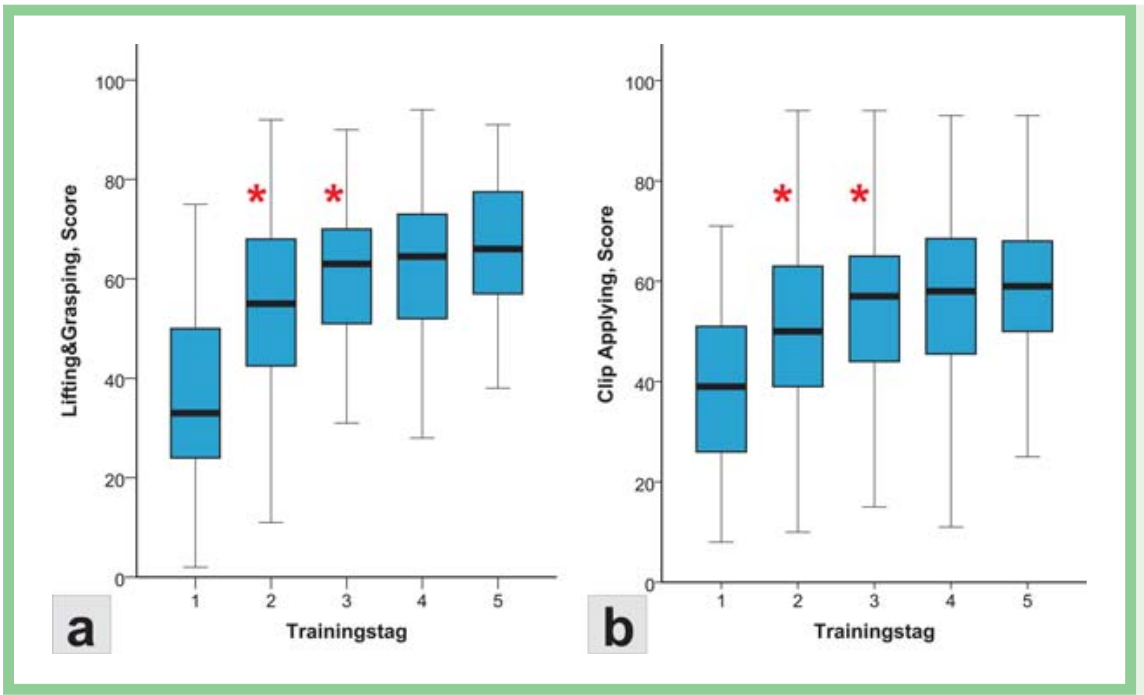

Abb. 2 Scores für die Übungen a „Lifting \& Grasping“ und b „Clip Applying“ über fünf Trainingstage. Signifikante Differenzen $(p<0,05)$ zum Vortag sind mit einem * markiert.

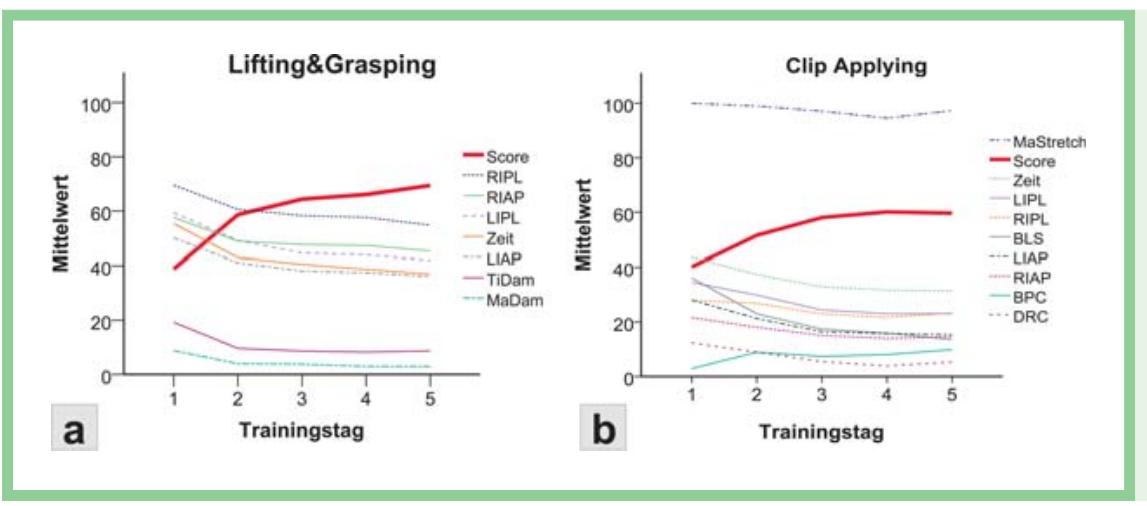

Abb. 3 Einzelparameter und errechnete Scores für die Übungen a „Lifting \& Grasping“ und b „Clip Applying“ über fünf Trainingstage (Abkürzungen für die Parameter, s. Tab. 1). Die Werte sind prozentual zur Bestleistung aller Teilnehmer eines Tages angegeben, um die gemeinsame Darstellung in einem Wertebereich zu ermöglichen.

folgten die Parameter TiDam, welcher die Gewebeschädigung durch fehlerhafte Berührung des Hintergrundes mit dem Instrument angibt, und MaDam, der die maximale Gewebeschädigung anzeigt. Bei diesen beiden Parametern lag eine weitere Streuung mit größerer Differenz zwischen Mittelwert und Bestleistung vor. Die Übung Clip-Applying (CA) stellte eine fortgeschrittene bimanuelle Aufgabe dar. Die Einzelparameter zeigten insgesamt ähnliche Verläufe zu LG. Die geringste Variabilität lag bei der Übungszeit vor, danach folgten die manuellen Parameter für die linke und rechte Hand. Der berechnete Blutverlust lag in einem ähnlichen Bereich, während verlorene und schlecht platzierte Clips deutlich niedrigere Werte zeigten.

Die Werte für die 5 Trainingstage wurden in 15 einzelnen Trainingssessions erhoben. Die Einzelwerte der Sessions zeigten dabei eine deutlich Rhythmik mit Leistungsspitzen und nachfolgendem Leistungsabfall ( $\bullet$ Abb.4). Bei näherer Betrachtung fand sich hierbei eine zirkadiane Rhythmik: Auffallend war jeweils ein Leistungstief in der ersten Session des Trainingstages im Vergleich zur zuletzt gemessenen Leistung. Es folgte dann eine erneute Leistungsverbesserung, die jeweils die Werte des Vortages übertraf. Auffällig war zudem ein stärker ausgeprägter Leistungsabfall zu Beginn des 4.Tages (Session 10, 0 Abb.4). Die Ursache hierfür war zunächst unklar. Bei genauer Betrachtung des Trainingsdatums der 10 . Session stellte sich jedoch heraus, dass dies der Morgen nach dem sog. „Ostseeabend“ war. Der Ostseeabend ist eine Veranstaltung, die von den meisten Kursteilnehmern besucht wird und meist bis später in den Abend reicht. Es zeigte sich also eine Leistungsabnahme am Folgetag nach mutmaßlich kürzerem Nachtschlaf.

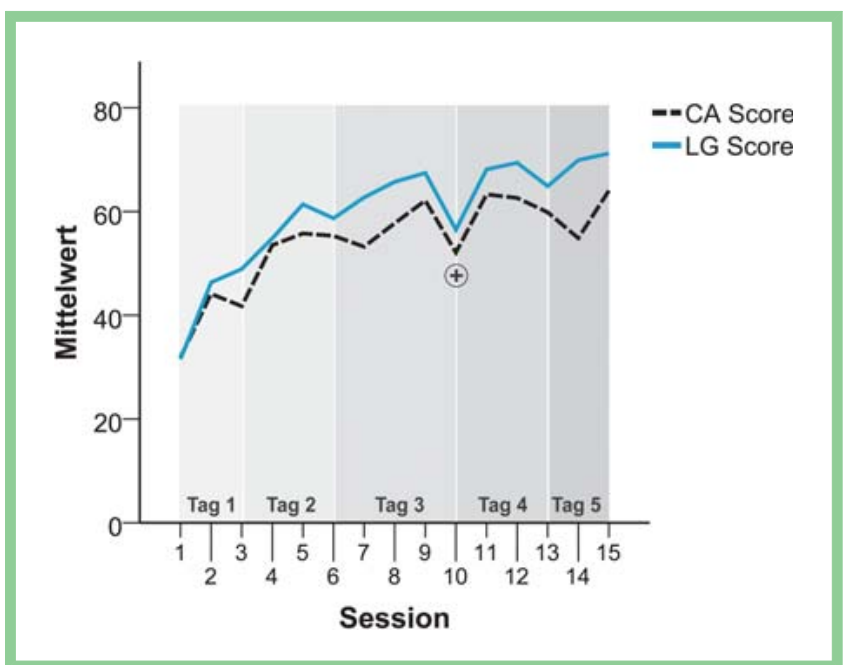

Abb.4 Scores für die einzelnen Trainingssessions (LG: „Lifting \& Grasping“; CA: „Clip Applying“). Die Probanden konnten die Sessions (S1-S15) über die zur Verfügung stehenden Tage verteilen. Die meisten Probanden führten dies wie folgt durch: S1 / 2: $\operatorname{Tag} 1 ;$ S3/4/ 5: $\operatorname{Tag} 2 ; 56 / 7 / 8 / 9$ : Tag 3; S10/11/ 12: Tag 4; S13/14/ 15: Tag 5. (+): Morgen nach dem „Ostseeabend“, einer Abendveranstaltung.

Um den Einfluss der chirurgischen Erfahrung der Teilnehmer auf die Simulatorleistung zu untersuchen, wurde eine Subgruppenanalyse anhand der im Eingangsfragebogen erhobenen laparoskopischen Erfahrung durchgeführt. Hierbei zeigte sich ein signifikanter Vorteil für die erfahrene Gruppe (>20 eigenstän- 

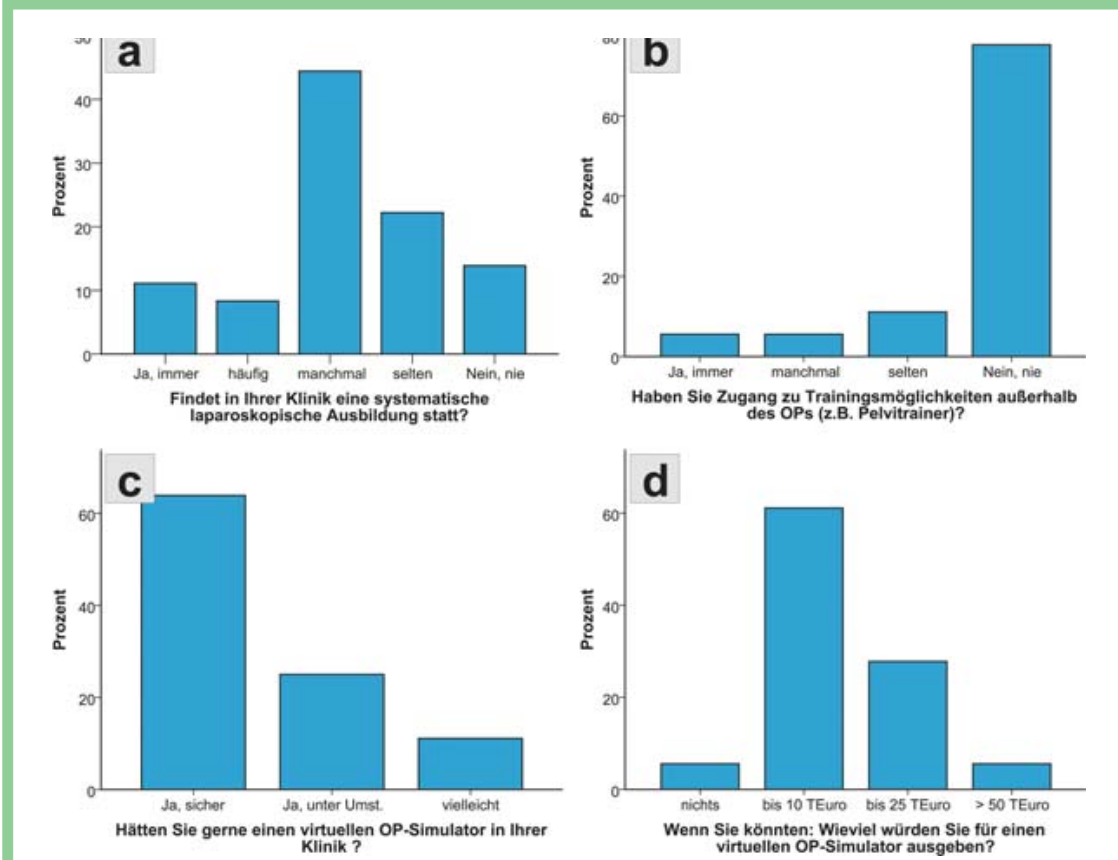

Abb.5 Teilnehmerbefragung. Antworten wurden mit einer 5-Punkte-Likert-Skala erfasst. Für Frage c wurden die Punkte 4 und 5 (kaum; nein) von keinem Teilnehmer angegeben, für Frage $\mathbf{d}$ wurde der Punkt 4 (bis 50 TEuro) von keinem Teilnehmer angegeben.

dige laparoskopische Operationen) gegenüber der unerfahrenen Gruppe ( $\leq 20$ lap. OPs) für die überwiegende Anzahl der Simulatorparameter sowie für die Scores ( $\bullet$ Tab.3). Die Parameter BPC (nicht korrekt platzierte Clips) und DRC (verlorene Clips) der Übung Clip Applying eigneten sich nicht für die Analyse, da die Teilnehmer hierbei im Median keine Fehler machten.

Am letzten Tag der Studie wurden Fragebögen ausgeteilt, mit denen Aspekte der chirurgischen Ausbildung der Teilnehmer und die Akzeptanz des Simulationstrainings erfragt wurden. Nur 19\% der Teilnehmer gaben an, dass in ihrer Klinik eine systematische laparoskopische Ausbildung stattfindet („immer“ bzw. „häufig“), während $81 \%$ diese Frage mit „manchmal“ bis „nie“ beantworte-

Tab.3 Simulatorleistung nach laparoskopischer Erfahrung für die Übungen „Lifting \& Grasping“ und „Clip Applying“.

\begin{tabular}{|c|c|c|c|}
\hline & $\begin{array}{l}\leq 20 \text { lap. OPs Median } \\
\text { (min-max) }\end{array}$ & $\begin{array}{l}\text { > } 20 \text { lap. OPs Median } \\
\text { (min - max) }\end{array}$ & $\mathbf{P}$ \\
\hline \multicolumn{4}{|c|}{ Lifting \& Grasping } \\
\hline Score & $56(4-91)$ & $63(2-94)$ & $\leq 0,001$ \\
\hline Zeit & $85,4(34,2-169)$ & $79,6(43,4-207)$ & 0,014 \\
\hline LIPL & $1,9(1,0-3,6)$ & $1,7(1,1-3,7)$ & 0,085 \\
\hline LIAP & $420,1(277-853)$ & $399,5(286-1076)$ & 0,003 \\
\hline RIPL & $1,7(1,17-2,8)$ & $1,6(1,0-2,8)$ & $\leq 0,001$ \\
\hline RIAP & $388,6(276-786)$ & $371,2(276-812)$ & 0,001 \\
\hline TiDam & $3(0-33)$ & $2(0-14)$ & 0,005 \\
\hline MaDam & $2,7(0-93,3)$ & $2,2(0-70)$ & 0,005 \\
\hline \multicolumn{4}{|c|}{ Clip Applying } \\
\hline Score & $51(8-92)$ & $56(12-94)$ & $\leq 0,001$ \\
\hline Zeit & $85,7(29,3-274)$ & $80,4(22,7-269)$ & 0,34 \\
\hline $\mathrm{BPC}$ & $0(0-3)$ & $0(0-3)$ & 0,971 \\
\hline DRC & $0(0-5)$ & $0(0-6)$ & 0,225 \\
\hline LIPL & $1,1(0,2-4,5)$ & $0,9(0,2-4,0)$ & $\leq 0,001$ \\
\hline LIAP & $209,3(38,5-1252)$ & $163,8(46,2-982)$ & $\leq 0,001$ \\
\hline RIPL & $1(0,2-4,5)$ & $1(0,2-4,7)$ & 0,722 \\
\hline RIAP & $201,9(33,2-1444)$ & $173(26,8-1130)$ & 0,094 \\
\hline MaStretch & $100(25,3-100)$ & $100(26,9-100)$ & 0,001 \\
\hline BLS & $0,3(0-2,0)$ & $0,2(0-2,0)$ & $\leq 0,001$ \\
\hline
\end{tabular}

ten ( $\bullet$ Abb.5a). In 78\% der Kliniken bestanden „nie“ Trainingsmöglichkeiten außerhalb des OPs ( $\bullet$ Abb.5b). 92\% antworteten auf die Frage, ob „die Ausbildungsqualität mit Simulatorsystemen gesteigert werden kann“ mit „Ja“ bzw. „Ja, unter Umständen“ (1 2 / Likert-5). Bezüglich der Frage, ob „Simulatoren Teile des konventionellen Trainings ersetzen können“, zeigte sich ein gemischtes Bild: $53 \%$ beantworteten dies mit „Ja“ bzw. „Ja, unter Umständen“ (1 - 2/ Likert-5). Auf die Frage, ob „Sie ein solches System gerne in Ihrer Klinik hätten“, antworteten 89\% mit 1 - 2/ Likert-5, $11 \%$ mit „vielleicht“ und keiner mit „kaum“ oder „nein“ ( $\bullet$ Abb. 5 c). Auf die Frage „Wenn Sie könnten: Wie viel Geld würden Sie für einen virtuellen OP-Simulator ausgeben?" antworteten 67\% mit „bis 10000 Euro“, $28 \%$ mit „bis 25000 Euro“, jedoch nur 6\% mit dem tatsächlichen Anschaffungspreis des verwendeten Simulators von „mehr als 50000 Euro“ ( $\bullet$ Abb.5d).

\section{Diskussion \\ $\nabla$}

Das Ziel der vorliegenden Studie war es, die Eignung von virtuellen laparoskopischen OP-Simulatoren zur Ausbildung in chirurgischen Trainingskursen zu untersuchen. Die Simulatoren sollten hierbei nicht in einem eigenständigen Simulatorkurs verwendet, sondern in ein bestehendes Kurssystem integriert werden, um ein reales Einsatzszenario zu erhalten. Die Simulatoren wurden daher im etablierten „Praktischen Kurs für Viszeralchirurgie“ in Warnemünde mit jährlich 108 Teilnehmern eingesetzt [9]. Mit der vorliegenden Studie sollten sowohl die Lernkurven am Simulator dargestellt werden, als auch die Eignung der so erhaltenen Trainingsparameter zur Leistungsbewertung eingeschätzt werden. Darüber hinaus wurde die Akzeptanz des Simulatortrainings bei den Teilnehmern erfragt, um Indikatoren für den praktischen Einsatz zu erhalten.

Die am Simulator gemessenen Leistungsscores zeigten den typischen Verlauf einer laparoskopischen Lernkurve [1, 14]. Die Übungsleistung stieg während der ersten beiden Tage - entsprechend sechs Trainingsdurchläufen - signifikant an. Danach zeigte sich ein langsamerer und nicht mehr signifikanter Leistungsan- 
stieg. Es ist anzunehmen, dass ab dem vierten Tag für die verwendeten Übungen eine Plateauphase erreicht wird, was allerdings nur durch eine noch länger andauernde Datenerfassung zu sichern wäre. Es kann jedoch festgestellt werden, dass der wesentliche Anteil der Lernkurve während des Kursverlaufes durchlaufen wurde. Ein Trainingseffekt am Simulator ist hierbei nicht sicher von einem Trainingseffekt durch den Kurs zu unterscheiden, da die Teilnehmer des Simulatortrainings parallel auch am normalen Kursverlauf teilgenommen haben. Dies war allerdings auch nicht das Ziel der Studie, da hier die Integration des Simulatortrainings in den chirurgischen Trainingskurs validiert werden sollte. Im Median trainierten die Teilnehmer knapp 10 Minuten täglich am Simulator. Im Vergleich zum normalen Kursprogramm mit mind. 4Stunden praktischem Training täglich ist dies vergleichweise wenig, so dass ein starker Effekt durch den Kurs zu vermuten ist. Es ist also anzunehmen, dass die gemessene Simulatorleistung zumindest teilweise den Trainingseffekt des Kurses reflektiert. Die Stärke dieses Effektes wäre allerdings nur durch eine Simulatorauswertung von Teilnehmern gleichen Erfahrungsgrades mit und ohne Kursteilnahme zu bestimmen, was über die Zielsetzung dieser Studie hinausgeht.

In den beiden Übungen wurden 16 einzelne Simulatorparameter erfasst. Um diese übersichtlich darzustellen, bildet die Simulatorsoftware daraus jeweils einen Score pro Übung ( $\bullet$ Tab. 1 ). Diese Scores werden zumeist als Grundlage der Leistungsbewertung benutzt. Allerdings ist die chirurgische Relevanz der Einzelparameter kaum untersucht, so dass die Gewichtung dieser Parameter zu Scores mit unterschiedlicher Aussagekraft führen kann. Neben der Beurteilung der errechneten Scores wurden daher auch die zugrunde liegenden Einzelparameter analysiert. Diese zeigten überwiegend einen harmonischen Anstieg, d.h., der Leistungszuwachs gestaltete sich prozentual für die erhobenen Parameter gleich stark ausgeprägt. Dies ist ein Hinweis darauf, dass für die meisten Parameter ein quantitativ ähnlicher Lerneffekt vorliegt. Diese Parameter eignen sich daher potenziell für die Leistungsbewertung im Rahmen des Kurses. In der Lifting \& Grasping-Übung lagen die manuell orientierten Parameter (Pfadlänge, Winkelpfad, Zeit) nahe beieinander, während der eher spezifisch-chirurgische Parameter der Gewebeschädigung (TiDam) prozentual deutlich niedriger lag ( $\bullet$ Abb.3). Ursächlich ist hier die höhere Leistungsvarianz durch die individuell verschiedene chirurgische Vorerfahrung der Teilnehmer. Einige Parameter (MaDam, BPC, DRC) waren aufgrund der gering ausgeprägten Leistungsunterschiede weniger zur Beurteilung geeignet. Dies zeigt, dass sich die vom Simulatorhersteller verwendeten Parameter zur Erstellung der Scores nicht gleich gut zur Leistungsbewertung eignen.

Bei der Auswahl von Parametern zur Leistungsbewertung können manuelle Basisparameter von fortgeschrittenen koordinativen bzw. übungsspezifischen Parametern unterschieden werden. Typische Basisparameter in der vorliegenden Studie waren die Pfadlänge und der Winkelpfad des rechten bzw. linken Instrumentes (RIPL, RIAP, LIPL, LIAP). Hierbei werden der zurückgelegte Weg der Instrumentenspitze bzw. die Winkelbewegungen gemessen. Diese Parameter beschreiben die sog. Bewegungsökonomie $[15,16]$. Bessere Werte deuten auf präzisere Bewegungen hin und sind ein typisches laparoskopisches Leistungsmerkmal. Ein fortgeschrittener Parameter in dieser Studie war die Gewebeschädigung. Übungsspezifische Parameter waren verlorene Clips oder Blutverlust. Mit den Scores werden die einzelnen Parameter rechnerisch zusammengefasst. Die Scores können dabei frei konfiguriert und den eigenen Bedürfnissen angepasst werden.
Für die fünf Trainingstage zeigte sich ein homogener lernkurventypischer Leistungszuwachs. Die Ergebnisse der einzelnen Trainingssessions (drei bis fünf Sessions/Tag) zeigten jedoch eine deutliche Rhythmik mit Leistungsspitzen und -senken. Insbesondere zeigte sich eine schlechtere Leistung jeweils zu Beginn des Trainingstages mit deutlicher Verbesserung im Tagesverlauf und in der Regel Erreichen eines Wertes über dem des Vortages. Eine mögliche Ursache hierfür könnte eine zirkadiane Rhythmik mit schlechterer morgendlicher Leistung sein. Wahrscheinlicher ist jedoch, dass eine gewisse Eingewöhnung zu Beginn des Tages erforderlich ist, was vermutlich auch der Realität im OP entspricht [17]. Dies unterstreicht aber auch die Notwendigkeit einer kumulativen Datenerfassung, sofern ein Simulator zur Leistungsbewertung benutzt werden soll. Ergebnisse einzelner Übungen sind hierzu also nicht aussagekräftig genug.

Im Trainingsverlauf der Studie war zudem ein akzentuierter Leistungsabfall zu Beginn des vierten Trainingstages auffällig. Dieser Leistungsabfall zeigte sich homogen für alle Parameter in beiden Übungen und war zunächst nicht erklärbar. Die nähere Betrachtung zeigte, dass einige Teilnehmer diesen Leistungsabfall nicht aufwiesen, so dass hier offenbar ein individueller Einfluss bestand. Es stellte sich schließlich überraschend ein externer Parameter als mutmaßliche Quelle heraus: Vor dem vierten Tag fand eine Abendveranstaltung als festlicher Höhepunkt des Kurses statt. Die Veranstaltung reichte für viele Teilnehmer bis spät in den Abend, so dass ein kürzerer Nachtschlaf die mutmaßliche Ursache der veränderten Leistung darstellt. Eine genaue Korrelation zur Teilnahme einzelner Probanden an der Abendveranstaltung konnte nicht hergestellt werden, da sich dieser Einfluss überraschend herausstellte und die Teilnahme an der Veranstaltung nicht im Rahmen der Studie statistisch erfasst wurde. Allerdings wurde dieses Defizit während des weiteren Trainingsverlaufes rasch aufgeholt. Dies zeigt aber auch, dass die manuelle und koordinative chirurgische Leistungsfähigkeit äußeren Einflüssen unterliegen kann. Ähnliche Ergebnisse, z. B. unter Schlafentzug oder nach Alkoholeinfluss, wurden bereits mehrfach beschrieben $[18,19]$. Mit einem OP-Simulator können solche Einflüsse direkt quantitativ untersucht werden.

Die hier verwendeten Übungen sind als Software vom Simulatorhersteller programmiert worden, orientieren sich jedoch an gängigen Übungsabläufen, die auch an anderen Simulatoren verwendet werden. Diesen Simulatorübungen ist teilweise eine Nähe zum herkömmlichen laparoskopischen Training gemeinsam. Zum Teil werden aber auch Übungen angeboten, die herkömmlich nicht oder nur schwer zu gestalten sind. Das Übungsdesign ist dabei an manuellen und koordinativen Fertigkeiten ausgerichtet, von denen man annimmt, dass diese für die Laparoskopie wichtig sind. Es handelt sich also um ein rein empirisches Übungsdesign, da keine formalen Kriterien existieren, um laparoskopische Trainingsabläufe zu beschreiben. Unter diesen Aspekten kann also nicht sichergestellt werden, dass das Simulatortraining auch tatsächlich die laparoskopischen Leistungen verbessert. So wäre es auch denkbar, dass sich nur die Leistung am Simulator selbst verbessert, ohne dass sich dies in der laparoskopischen Praxis widerspiegelt. Um diesen Aspekt näher zu betrachten, wurde in der vorliegenden Studie eine Subgruppenanalyse von erfahrenen Teilnehmern (mehr als 20 eigenständige laparoskopische Operationen) gegen weniger erfahrene (weniger als 20 Operationen) durchgeführt. Es wurde also die sog. Konstruktvalidität des Simulators untersucht, die eine statistisch signifikante Differenz zwischen Gruppen verschiedener Erfahrungsgrade beschreibt [4]. Hierbei zeigte sich ein höchst signifi- 
kanter Leistungsvorteil für die erfahrene Gruppe $(\mathrm{p} \leq 0,001)$ in den Scores beider Übungen. Dies deutet darauf hin, dass der Simulator Leistungen trainiert und misst, die tatsächlich laparoskopisch relevant sind. Allerdings zeigte sich diese Differenz nicht in allen Einzelparametern, so dass einzelne Parameter ggf. für eine Leistungsbewertung ausgeschlossen werden können. Für eine definitive Beurteilung einzelner Parameter wären allerdings eine höhere Teilnehmerzahl und weitere Erfahrungsgruppen erforderlich. Zudem ist dies vom Kursumfeld, insbesondere der vorliegenden Erfahrung der Teilnehmer abhängig.

Zum Abschluss der Studie wurden Fragen in Bezug auf die Ausbildungssituation der Teilnehmer und die Akzeptanz des Simulatortrainings gestellt. Hierbei zeigte sich, dass nur in wenigen Kliniken eine systematische laparoskopische Ausbildung stattfindet. Regelmäßige Trainingsmöglichkeiten außerhalb des OPs waren mit nur etwa 20\% die Ausnahme. Die überwiegende Anzahl der Teilnehmer hat vor Ort also weder eine strukturierte laparoskopische Ausbildung, noch Zugriff auf entsprechende Trainingsmöglichkeiten. Dies unterstreicht die schwierige Ausbildungssituation in Deutschland und betont die Bedeutung eines strukturierten Trainings auch außerhalb der eigenen Klinik [8]. Simulatoren können hierzu in besonderer Weise beitragen, da das Simulatortraining prinzipiell nicht orts- und zeitgebunden ist. Mit Simulatoren kann ein strukturiertes Training durch vorgegebene Übungen mit überprüfbarer Leistung erreicht werden. Mehr als $90 \%$ der Teilnehmer gaben an, dass mit Simulatoren die Ausbildungsqualität gesteigert werden kann und fast 90\% hätten gerne einen Simulator an ihrer Klinik.

Auf die Frage nach der Anschaffung eines Simulators würden zwei Drittel der Teilnehmer nur bis 10000 Euro ausgeben. Den realen Preis von mindestens 50000 Euro würden nur 6\% bezahlen. Dies zeigt zum Einen das finanzielle Dilemma der außerklinischen chirurgischen Ausbildung, die durch Kursbeiträge allein kaum zu finanzieren ist [9]. Zum Anderen scheint das Simulatortraining noch nicht einen Stellenwert erreicht zu haben, der die hohen Anschaffungskosten rechtfertigt. Diese Kosten waren auch ein Grund für die limitierte Teilnahme von 36 Teilnehmern an der vorliegenden Studie, da eine höhere Teilnehmerzahl wesentlich mehr Geräte erfordert hätte. Für den tatsächlichen Einsatz im Kursalltag wäre ein Simulator für je zwei Teilnehmer erforderlich, was bei 108 Kursteilnehmern Anschaffungskosten von etwa 5 Millionen Euro bedeuten würde und damit nicht realisierbar ist. Für den praktischen Einsatz im Kursrahmen wären jedoch alternative Modelle mit kürzeren Trainingszeiten und wechselnder Besetzung denkbar.

\section{Schlussfolgerung}

\section{V}

Die Lernkurve am virtuellen laparoskopischen Simulator wurde während des Kursverlaufes in fünf Trainingstagen durchlaufen. Es zeigte sich eine deutliche Leistungsverbesserung mit nachfolgender Plateauphase. Die Simulatorscores konnten unterschiedliche Erfahrungsgrade statistisch eindeutig differenzieren, so dass die Konstruktvalidität des Simulators bestätigt werden konnte. Damit eignet sich der Simulator auch zur Leistungsbewertung im Kursrahmen. Es zeigte sich eine größere Variabilität in Bezug auf die zugrunde liegenden Einzelparameter, was eine Anpassung der Scores für den eigenen Anwendungsbereich gestattet. So kann ein Fokus auf manuelle Basisparameter für einen Anfängerkurs sinnvoll sein, während sich übungsspezifische Parameter für Fortgeschrittene besser eignen. Ein interessanter
Nebenaspekt war der Effekt eines kürzeren Nachtschlafes während des Kursverlaufes, der zu vorübergehend schlechteren Leistungen führte. Die Befragung der Studienteilnehmer zeigte eine schwierige Ausbildungssituation. Das Simulatortraining wurde sehr gut akzeptiert. Allerdings schätzte die Mehrzahl der Teilnehmer die Kosten der Geräte falsch ein, was auch ein genereller Parameter für die Einschätzung der Ausbildungskosten sein mag. Virtuelle OP-Simulatoren besitzen spezifische Vorteile wie flexible Trainingszenarien und umfangreiche Bewertungsmöglichkeiten. Mit den derzeitigen Preisen der Geräte sind Simulatoren für den breiten praktischen Einsatz in einem großen Kurs jedoch kaum finanzierbar.

\section{Interessenkonflikt: Nein}

\section{Literatur}

1 Lehmann KS, Ritz JP, Maass $\mathrm{H}$ et al. A prospective randomized study to test the transfer of basic psychomotor skills from virtual reality to physical reality in a comparable training setting. Ann Surg 2005; 241: $442-449$

2 Rogers DA, Elstein AS, Bordage G. Improving continuing medical education for surgical techniques: applying the lessons learned in the first decade of minimal access surgery. Ann Surg 2001; 233: 159-166

3 Palter VN, Grantcharov TP. Virtual reality in surgical skills training. Surg Clin North Am 2010; 90: 605-617

4 Thijssen AS, Schijven MP. Contemporary virtual reality laparoscopy simulators: quicksand or solid grounds for assessing surgical trainees? Am J Surg 2010; 199: 529-541

5 Gurusamy KS, Aggarwal R, Palanivelu L et al. Virtual reality training for surgical trainees in laparoscopic surgery. Cochrane Database Syst Rev 2009: CD006575

6 Seymour NE, Gallagher AG, Roman SA et al. Virtual reality training improves operating room performance: results of a randomized, doubleblinded study. Ann Surg 2002; 236: 458-463

7 Healy GB. The College should be instrumental in adapting simulators to education. Bull Am Coll Surg 2002; 8: 10-12

8 Gröne J, Ritz J-P, Stroux A et al. Measurable learning effects after a 1-week skills course in digestive surgery Int J Colorectal Dis 2010; 25: $1133-1139$

9 Ritz J-P, Gröne J, Hopt U et al. "Practical course for visceral surgery in Warnemünde" 10 years on. Significance and benefits of a surgical training course. Chirurg 2009; 80: 864-871

10 Fairhurst $K$, Strickland A, Maddern G. The LapSim virtual reality simulator: promising but not yet proven. Surg Endosc 2010; 25: 343 - 355

11 Hyltander A, Liljegren E, Rhodin PH et al. The transfer of basic skills learned in a laparoscopic simulator to the operating room. Surg Endosc 2002; 16: 1324-1328

12 van Dongen KW, Tournoij E, van der Zee DC et al. Construct validity of the LapSim: can the LapSim virtual reality simulator distinguish between novices and experts? Surg Endosc 2007; 21: 1413-1417

13 Panait $L$, Bell RL, Roberts KE et al. Designing and validating a customized virtual reality-based laparoscopic skills curriculum. J Surg Educ 2008; 65: 413-417

14 Moore MJ, Bennett CL. The learning curve for laparoscopic cholecystectomy. The Southern Surgeons Club. Am J Surg 1995; 170: 55-59

15 Van Nortwick SS, Lendvay TS, Jensen AR et al. Methodologies for establishing validity in surgical simulation studies. Surgery 2010; 147 : $622-630$

16 Grantcharov TP, Rosenberg J, Pahle E et al. Virtual reality computer simulation. Surg Endosc 2001; 15: 242 - 244

17 Calatayud D, Arora S, Aggarwal R et al. Warm-up in a virtual reality environment improves performance in the operating room. Ann Surg 2010; 251: $1181-1185$

18 Dawson D, Reid K. Fatigue, alcohol and performance impairment. Nature 1997; 388: 235-235

19 Lehmann KS, Martus P, Little-Elk S et al. Impact of sleep deprivation on medium-term psychomotor and cognitive performance of surgeons: Prospective cross-over study with a virtual surgery simulator and psychometric tests. Surgery 2010; 147: 246-254 